\title{
PROPIEDADES PSICOMÉTRICAS DEL CUESTIONARIO DE RIESGOS PSICOSOCIALES COPSOQ-ISTAS 21 Y APLICACIÓN EN DOCENTES UNIVERSITARIOS ARGENTINOS"
}

\author{
Lucas Pujol-Cols \\ Mariana Arraigada ${ }^{* * * *}$
}

* doi: 10.11144/Javeriana.cao30-55.ppcr. Este artículo es el resultado de la investigación 15/H244 con financiamiento de la Universidad Nacional de Mar del Plata, Argentina. El artículo se recibió el 10/07/2017 y se aprobó el 25/11/2017. Sugerencia de citación: Pujol-Cols, L., y Arraigada, M. (2017). Propiedades psicométricas del Cuestionario de Riesgos Psicosociales Copsoq-Istas 21 y aplicación en docentes universitarios argentinos. Cuadernos de Administración, 30(55), 97-125. http://dx.doi.org/10.11144/Javeriana. cao30-55.ppcr.

** Candidato a Doctor en Administración, Universidad Nacional del Centro de la Provincia de Buenos Aires, Argentina. Magíster en Administración de Negocios, Universidad Nacional de Mar del Plata, Mar del Plata, Argentina. Investigador Asociado, Vicerrectoría de Investigación y Postgrado, Universidad Católica del Maule, Curicó, Chile. Becario Doctoral, Consejo Nacional de Investigaciones Científicas y Técnicas (CONICET), Mar del Plata, Argentina.

Correo electrónico: lucaspujolcols@gmail.com

*** Magíster en Administración de Negocios, Universidad Nacional de Mar del Plata, Argentina. Profesora Adjunta regular, Facultad de Ciencias Económicas y Sociales, Universidad Nacional de Mar del Plata, Mar del Plata, Argentina.

Correo electrónico: marianaarraigada@gmail.com 


\section{Propiedades \\ psicométricas del \\ Cuestionario de Riesgos \\ Psicosociales Copsoq- Istas 21 y aplicación en docentes universitarios argentinos}

\section{RESUMEN}

En este artículo se examinan, primero, las propiedades psicométricas de la versión corta del Copsoq-Istas 21 (1.5) y, segundo, la prevalencia de seis factores de riesgo psicosocial, en una muestra de 175 docentes universitarios argentinos. Los resultados revelan que el instrumento observa una confiabilidad aceptable, con excepción de la sub-escala de doble presencia. Si bien los hallazgos de este estudio brindan soporte a un modelo compuesto por seis factores latentes, la obtención de resultados poco satisfactorios en las pruebas de validez convergente y discriminante sugiere la necesidad de rediseñar el instrumento con el propósito de mejorar su validez de constructo. Finalmente, los análisis practicados revelan una prevalencia elevada de doble presencia, exigencias psicológicas e insuficiente estima en los docentes participantes.

\section{Palabras clave: riesgos psicosociales, validación, propiedades psicométricas, docentes universitarios, educación superior. \\ Clasificación JEL: Co1, Ioo, M12}

\section{Psychometric properties \\ of the Copsoq-Istas \\ Psychosocial Risk \\ Questionnaire 21 in \\ Argentinian university teachers}

\section{ABSTRACT}

This article examines the psychometric properties of the short version of the Copsoq-Istas 21 (1.5) and analyzes the prevalence of six work-related psychosocial risk factors in a sample of 175 Argentinian university teachers. The results reveal that the Copsoq-Istas 21 (1.5) shows acceptable reliability, except for the double presence sub-scale. Furthermore, although the results of this study provide support to a six-factor structure of the Copsoq-Istas 21 (1.5), dissatisfactory results in the convergent and discriminant validity tests suggest the need to re-design some of the sub-scales in order to improve the overall construct validity of the instrument. Finally, the findings also show a high prevalence of double presence, psychological demands, and insufficient esteem affecting the teachers who participated in the study.

Keywords: Psychosocial risks, validation, psychometric properties, teachers, higher education.

JEL Classification: $\mathrm{Col}$, Ioo, M12

\section{Propriedades \\ psicométricas do \\ Questionário de Riscos \\ Psicossociais Copsoq- \\ Istas 21 e aplicação em docentes universitários argentinos}

\section{RESUMO}

Neste artigo, examinam-se, primeiro, as propriedades psicométricas da versão curta do Copsoq-Istas 21 (1.5) e, segundo, a prevalência de seis fatores de risco psicossocial, numa amostra de 175 docentes universitários argentinos. Os resultados revelam que o instrumento observa uma confiabilidade aceitável, com exceção da subescala de dupla presença. Embora os achados deste estudo ofereçam suporte a um modelo composto por seis fatores latentes, a obtenção de resultados pouco satisfatórios nos testes de validade convergente e discriminante sugere a necessidade de redesenhar o instrumento com o propósito de melhorar sua validade de construto. Finalmente, as análises praticadas revelam uma prevalência elevada de dupla presença, exigências psicológicas e insuficiente estima nos docentes participantes.

Palavras-chave: riscos psicossociais, validação, propriedades psicométricas, docentes universitários, educação superior. Classificação JEL: Co1, loo, M12 


\section{Introducción}

El sistema universitario a nivel mundial ha experimentado profundas transformaciones en las últimas décadas, entre las que se destacan niveles crecientes de interdependencia entre las entidades educativas; una adaptación cada vez mayor de las decisiones, comportamientos y prácticas internas a los requerimientos del mercado; niveles cada vez más feroces de competencia entre las entidades educativas por la obtención de recursos físicos, financieros y humanos; y una preocupación cada vez mayor por la eficiencia en las operaciones y la rendición de cuentas (Blaschke, Frost y Hattke, 2014; Bradshaw y Fredette, 2009). Estos cambios se han traducido en nuevas presiones y desafíos para los académicos en las universidades, entre los que se incluyen requerimientos más elevados de productividad científica, formación académica y pedagógica e innovación tecnológica (García, Iglesias, Saleta y Romay, 2016; Unda, Uribe, Jurado, García, Tovalín y Juárez, 2016; Watts y Robertson, 2011). En este sentido, numerosos autores reconocen que las elevadas demandas cognitivas (las que se derivan del desempeño de tareas complejas, que requieren altos niveles de concentración y precisión) y emocionales (las que emergen de los requerimientos de interacción y vinculación emocional con diferentes actores, tales como estudiantes, directivos, colegas y subordinados) a las que deben hacer frente los académicos durante el ejercicio de su rol han convertido la profesión académica en una ocupación muy estresante (Fredman y Doughney, 2012; Kinman y Wray, 2013; Llorens, Cifre, Salanova y Martínez, 2003a; Llorens, García-Renedo, Salanova y Cifre, 2003b; Mudrak et ál., 2017; Salanova, Martínez y Lorente, 2005).

Idealmente, los profesionales insertos en ocupaciones demandantes, como lo es la profesión académica, deberían contar con elevados recursos laborales, entendidos como aquellos factores físicos, psicológicos, organizacionales o sociales del trabajo que son relevantes para lograr los objetivos; son funcionales para reducir las demandas laborales, así como sus costos fisiológicos y psicológicos asociados; y estimulan el crecimiento personal, el aprendizaje y el desarrollo de los empleados (Bakker y Demerouti, 2014). A pesar de esto, numerosas investigaciones previas reconocen que los docentes universitarios suelen sentir que el nivel que reciben de algunos recursos laborales fundamentales, tales como el reconocimiento, el apoyo social de colegas y supervisores y las compensaciones monetarias, resultan insuficientes (Kinman y Wray, 2013; Fredman y Doughney, 2012), situación que eleva los riesgos psicosociales, entendidos como aquellos factores relacionados con el trabajo que, sea por exceso, defecto o combinación, constituyen una amenaza para la integridad física, social y/o psicológica de los individuos, a los que se hallan expuestos los académicos (Meliá et ál., 2006). 
El estudio de los factores del trabajo que exponen a los docentes universitarios a mayor riesgo psicosocial, así como la identificación de los grupos más vulnerables, son indispensables para el desarrollo de políticas y estrategias de gestión que faciliten su retención y desarrollo y que, a la vez, preserven su salud física y mental (Botero, 2012; Manassero, Vázquez, Ferrer, Fornés, Fernández y Queimadelos, 2003; Unda, Sandoval y Gil-Monte, 2007). Sin embargo, y a pesar de constituir un tema altamente relevante, tal y como señalan Unda et ál. (2016), las investigaciones focalizadas en el estudio de los riesgos psicosociales, así como en la construcción y validación de instrumentos que habiliten su evaluación, aún resultan limitadas en Latinoamérica. De este modo, y con el principal propósito de contribuir al avance del conocimiento en materia de riesgos psicosociales en docentes universitarios, el presente artículo persigue dos objetivos fundamentales. El primero consiste en examinar las propiedades psicométricas del Cuestionario para la Evaluación de Riesgos Psicosociales en el Trabajo Copsoq-Istas 21 (versión 1.5). El segundo consiste en medir la prevalencia de seis categorías genéricas de riesgos psicosociales (exigencias psicológicas, control sobre el trabajo, apoyo social y calidad del liderazgo, doble presencia, estima e inseguridad sobre el futuro) en una muestra de docentes pertenecientes a una universidad pública argentina y, luego, analizar la influencia de un conjunto de variables sociodemográficas y laborales sobre los niveles de exposición reportados por los participantes.

\section{Los riesgos psicosociales en el trabajo}

Bajo la premisa que la salud es mucho más que un mero estado de ausencia de afección o desequilibrio (Salanova, 2008), la Psicología Organizacional pareciera estar adoptando poco a poco una aproximación menos centrada en el diagnóstico de la enfermedad y más enfocada en el estudio preventivo de las condiciones laborales que devienen en estados individuales y organizacionales saludables (Salanova, Martínez y Llorens, 2014). Esta aproximación teórica se sitúa dentro de la denominada Psicología Organizacional Positiva (Seligman y Csikszentmihalyi, 2000), definida como el estudio científico del funcionamiento óptimo de las personas y de los grupos en las organizaciones, así como su gestión efectiva (Schaufeli, Salanova, González-Roma y Bakker, 2002; Salanova, Martínez y Llorens, 2005). En efecto, y puesto que la experimentación de estados negativos en el trabajo suele ser la excepción más que la norma (Hakanen, Bakker y Schaufeli, 2006), la misma ha emergido como una perspectiva útil para explicar con mayor efectividad diversos fenómenos de interés para el Comportamiento Organizacional (Luthans, 2002). 
Los profundos cambios que se han producido en el mundo del trabajo en los últimos años, como resultado de procesos intensos de internacionalización y liberación económica, privatización y avances científico-tecnológicos (Parker, Wall y Cordery, 2001; Unda et ál., 2016), parecieran haberse materializado en nuevas demandas para los trabajadores, situación que ha incrementado la preocupación de académicos, directivos, funcionarios y representantes sindicales por mejorar las condiciones laborales en las organizaciones en un intento por preservar la salud física y mental del capital humano. En efecto, el diseño del trabajo (esto es, las tareas que involucra y las condiciones físicas, sociales y organizacionales para llevarlas a cabo) expone a los empleados a diversidad de riesgos psicosociales que, de no ser enfrentados de manera exitosa, son proclives a conducir a la experimentación de distrés y, en consecuencia, a provocar trastornos psicológicos, fisiológicos y conductuales en los individuos (Unda et ál., 2016).

Para Meliá et ál. (2006), los factores de riesgo psicosocial en el trabajo se refieren a aquellas demandas relacionadas con el trabajo que, sea por exceso, defecto o combinación, constituyen una amenaza para la integridad física, social y/o psicológica de los individuos. Pero, como advierten Schaufeli y Salanova (2002), los riesgos psicosociales no sólo emergen de la presencia de demandas laborales excesivas, sino que, también, aparecen cuando los recursos laborales disponibles para enfrentarlas son percibidos como insuficientes. Desde esta postura, y con sustento en el Modelo de Recursos y Demandas Laborales (Demerouti, Bakker, Nachreiner y Schaufeli, 2001), Schaufeli y Salanova (2002) proponen que los riesgos psicosociales se pueden dividir en dos grandes categorías. Por un lado, se encuentran las demandas laborales, las cuales refieren a aquellos aspectos físicos, sociales y organizacionales que le requieren al empleado un esfuerzo sostenido y están asociados a ciertos costos fisiológicos y psicológicos (p. ej. la sobrecarga cuantitativa, el conflicto de rol). Por el otro, se encuentra la falta de recursos laborales, entendidos como aquellos aspectos físicos, psicológicos, sociales y organizacionales del trabajo que son funcionales en la consecución de las metas, reducen las demandas laborales y estimulan el crecimiento y desarrollo personal y profesional (p. ej. el reconocimiento, el apoyo social).

Entender los riesgos psicosociales como una amenaza proveniente de la organización del trabajo, que tiene su origen en la presencia de elevadas demandas laborales y/o en la insuficiencia de recursos organizacionales, no implica afirmar que el trabajo sea en sí mismo patógeno o que la mera exposición a factores de riesgo conduce necesariamente a la experimentación de distrés u otros estados negativos en los empleados (Schaufeli y 
Salanova, 2002). Por el contrario, la literatura señala que la relación entre los factores psicosociales laborales y sus consecuencias fisiológicas, psicológicas y conductuales se ven moderadas por un conjunto de recursos personales (ver el concepto de capital psicológico positivo en Avey, Reichard, Luthans y Mhatre, 2011) que contribuyen a amortiguar los efectos negativos de los estresores y del distrés laboral sobre la salud ocupacional (Xanthopoulou, Bakker, Demerouti y Schaufeli, 2007).

De la gran cantidad de modelos sobre factores laborales de riesgo psicosocial que existen en la literatura, Neffa (2015) analiza y sintetiza cuatro de ellos. El primero de estos modelos es desarrollado por Karasek (1979), quien propone que el distrés laboral se genera como resultado de una combinación de trabajos con demandas psicológicas elevadas y un reducido margen de decisión. El segundo, llamado Modelo de desequilibrio entre la intensidad de la demanda de trabajo y la recompensa recibida (Siegrist, 1996), sostiene que la experiencia de distrés emerge de una percepción de incongruencia o desbalance entre los esfuerzos realizados y las recompensas recibidas a cambio. El tercero, denominado Modelo de justicia organizacional (Moorman, 1991), surge de la Teoría de la equidad y propone que los individuos desarrollan creencias acerca de lo que consideran justo 0 injusto en las relaciones, los procedimientos y la distribución de los recursos, como contrapartida del trabajo realizado. Este último modelo argumenta que la percepción de violación de las normas de justicia dentro de las organizaciones representa la causa principal del deterioro en la salud psíquica y física de los trabajadores. Finalmente, y con sustento en los tres modelos anteriores, un equipo de investigadores del Instituto Sindical de Trabajo, Ambiente y Salud de España proponen el Modelo ISTAS, desde el cual definen los factores psicosociales como aquellos factores de riesgo para la salud que se originan en la organización del trabajo y que generan respuestas de tipo fisiológico, emocional, cognitivo y conductual en los empleados (Moncada et ál., 2004).

\section{Evaluación de los riesgos psicosociales en el trabajo}

El carácter subjetivo de los riesgos psicosociales en el trabajo no impide que se los mida y evalúe (Neffa, 2015). De hecho, su medición periódica brinda a los directivos de las organizaciones información útil para diseñar estrategias preventivas que coadyuven a preservar la salud física y mental de sus empleados. Si bien algunos estudios han recurrido al empleo de fuentes cualitativas de información ( $p$. ej. análisis de puestos, entrevistas semiestructuradas, etc.), el enfoque predominante en la medición y evaluación de los riesgos psicosociales es el cuantitativo, sustentado en las metodologías de la psicometría (Meliá et ál., 2006). A tales fines, una gran cantidad de cuestionarios de 
auto-informe sobre riesgos psicosociales han sido desarrollados y validados en múltiples contextos laborales (ver Charria, Sarsosa y Arenas, 2011 para una revisión). Entre los instrumentos que predominan en la literatura internacional se distinguen el Cuestionario sobre la Experiencia y Evaluación del Trabajo (Questionnaire on the Experience and Assessment of Work, VBBA; Van Veldhoven et ál., 1997), el Cuestionario de Contenido del Trabajo (Job Content Questionnaire, JCQ; Karasek et ál., 1998), el Cuestionario del Desequilibrio Esfuerzo-Recompensa (Effort-Reward Imbalance Questionnaire; Siegrist, 1996) y el Cuestionario Psicosocial de Copenhague (Copenhagen Psychosocial Questionnaire, CoPsoQ; Kristensen et ál., 2005; Pejtersen et ál., 2010).

Dirigiendo la atención específicamente al ámbito iberoamericano, entre los instrumentos más difundidos se destacan el Cuestionario de Evaluación de Riesgos Psicosociales (30 ítems), construido por el Instituto Navarro de Salud Laboral (2002); la Batería de Riesgos Psicosociales (251 ítems), desarrollada conjuntamente por la Pontificia Universidad Javeriana y el Ministerio de Protección Social (2010) de Colombia; el Cuestionario de Condiciones de Trabajo (44 ítems), de Blanch, Sahagún y Cervantes (2010); y el Cuestionario para la Evaluación de Riesgos Psicosociales en el Trabajo Copsoq-Istas 21 (Moncada et ál., 2005), adaptación del Cuestionario Psicosocial de Copenhague (Kristensen et ál., 2005). Este último constituye uno de los instrumentos más empleados en Iberoamérica en la evaluación de los riesgos psicosociales en el trabajo (García, Iglesias, Saleta y Romay, 2016) y ha sido específicamente diseñado para relevar el nivel de exposición de los empleados a seis factores de riesgo (tres demandas y tres recursos laborales) con fuertes implicaciones para su bienestar psicosocial: las exigencias psicológicas del trabajo, el control sobre el trabajo, el apoyo social y calidad del liderazgo, la inseguridad sobre el futuro, la doble presencia y la estima.

Entre las razones que sustentan la elección del Copsoq-Istas como uno de los más empleados tanto en la investigación científica como en la práctica profesional se encuentran su exhaustividad (puesto que incluye una amplia variedad de factores psicosociales de riesgo, los cuales emergen de una síntesis e integración de teorías previas), la simplicidad en su administración y en la interpretación de sus resultados (en la medida en que cuenta con manuales para la autoadministración y la tabulación de las respuestas) y la flexibilidad en su aplicación (al admitir su adaptación a diversidad de contextos laborales y categorías ocupacionales). La edición 1.5 del mismo se presenta en tres extensiones: corta (de 38 ítems), media (de 99 ítems) y larga (de 133 ítems) y ha sido aplicada en distintos países de Latinoamérica, incluyendo a México (García et ál., 2016), Chile (Alvarado et ál., 2009; Alvarado et ál., 2012) y Argentina (Acevedo, Sánchez, Farías y 
Fernández, 2013). En particular, y en virtud de su corta extensión, la versión reducida del Copsoq-Istas 21 resulta prometedora para la investigación pues facilita su administración en conjunto con otras escalas en diseños metodológicos complejos que se focalicen en el estudio de los riesgos psicosociales en su relación con otros fenómenos de interés (como actitudes, intenciones y/o conductas).

\section{Riesgos psicosociales en docentes universitarios en Iberoamérica}

Las elevadas demandas cognitivas y emocionales a las que deben hacer frente los docentes universitarios durante el devenir de su carrera han convertido la profesión académica en una de las más estresantes (Kinman y Wray, 2013; Mudrak et ál., 2017; Salanova et ál., 2005; Fredman y Doughney, 2012). En una investigación documental, Botero (2012) identifica diversos factores del trabajo que parecieran afectar a los docentes universitarios latinoamericanos. Entre aquellos se encuentran: la multiplicidad de tareas, la sobrecarga de trabajo, el escaso tiempo disponible para cumplir con las actividades académicas, la falta de reconocimiento, la elevada carga mental, los espacios físicos de trabajo inadecuados, la falta de una remuneración que compense apropiadamente los esfuerzos y el escaso apoyo social de colegas y supervisores.

En un estudio realizado por Rodríguez, Oramas y Rodríguez (2007), los autores identifican la multiplicidad de funciones, las condiciones físicas inadecuadas, las reducciones presupuestarias, las escasas oportunidades de promoción, las relaciones conflictivas con alumnos y compañeros de trabajo y el insuficiente apoyo social y liderazgo como los principales factores psicosociales de riesgo a los que se encuentran expuestos los docentes. En otra investigación, García et ál. (2016) revelan una elevada prevalencia de exigencias psicológicas, insuficiente estima, doble presencia, inseguridad laboral y escaso apoyo social y liderazgo en una muestra de 621 académicos españoles. En otro estudio, en el que se contó con la participación de 500 académicos provenientes de distintas universidades públicas mexicanas, Unda et ál. (2016) reportan que las dificultades involucradas en las relaciones con los estudiantes, en conjunto con un acceso limitado a recursos físicos, constituyen los principales riesgos psicosociales a los que se encuentran expuestos los académicos participantes. En lo que refiere al contexto de las universidades argentinas, y si bien algunos trabajos han procurado describir las características del trabajo docente desempeñado en su interior (García de Fanelli y Moguillansky, 2014), no se encontraron investigaciones previas publicadas en las que se haya examinado la prevalencia de categorías genéricas de riesgos psicosociales en 
docentes universitarios argentinos, menos aún la influencia de variables sociodemográficas y laborales en tales niveles de exposición.

\section{Método}

\section{Participantes}

Se contó con una muestra de 175 docentes de una universidad argentina, seleccionados de manera aleatoria, cuya edad osciló entre 23 y 70 años, con una media de 44,72 y una desviación estándar de 11,85. Cerca del 71\% de los participantes eran mujeres, el $38,86 \%$ eran profesores y el $24,57 \%$ contaba con un contrato de tiempo completo. La antigüedad de los docentes osciló entre 1 y 45 años, con una media de 18,04 y una desviación estándar de 10,38. La antigüedad en el cargo actual varió entre 1 y 30 años, con una media de 8,88 y una desviación estándar de 8,44 . Cerca del $69 \%$ de los participantes tenían título de Grado, el 20\% título de Máster y el 11\% restante título de Doctor.

\section{Instrumento}

Se utilizó la versión reducida del Cuestionario de Evaluación de Riesgos Psicosociales en el Trabajo Copsoq-Istas 21 (1.5) de Moncada et ál. (2005), el cual resulta ser una adaptación del Cuestionario Psicosocial de Copenhague (Kristensen et ál., 2005). Este instrumento se encuentra compuesto por 38 reactivos y ha sido específicamente diseñado para identificar, medir y valorar la exposición de los empleados a seis grandes grupos de factores de riesgo para la salud en el trabajo de naturaleza psicosocial: (a) exigencias psicológicas (las demandas que emergen de la relación entre el volumen de trabajo y el tiempo disponible para realizarlo, así como de las exigencias de no involucrarse en la situación emocional que se deriva de las relaciones interpersonales laborales), (b) control sobre el trabajo (el grado en que el trabajo le provee al empleado de oportunidades de autonomía y desarrollo), (c) inseguridad sobre el futuro (la preocupación sostenida del empleado por una eventual pérdida de empleo o por la posibilidad de cambios no deseados en las condiciones de trabajo fundamentales), (d) apoyo social y calidad del liderazgo (la medida en la que el trabajo le permite al empleado el desarrollo de vínculos sociales y de colaboración con sus superiores y pares), (e) doble presencia (la dificultad experimentada por el empleado para balancear las demandas de su trabajo y las provenientes del dominio doméstico-familiar) y ( $f$ ) estima (el grado en que el empleado percibe que el respeto, el reconocimiento y el trato que recibe resultan 
apropiados en comparación con el esfuerzo invertido en su trabajo). Cada una de las seis dimensiones del Copsoq-Istas 21 incluye una escala de respuesta de cinco puntos, con valores ubicados entre el 0 (ninguna exposición al factor, salvo puntaje inverso) y el 4 (total exposición al factor, salvo puntaje inverso).

\section{Procedimiento}

Este artículo es parte de un proyecto de investigación más amplio focalizado en el estudio de los riesgos psicosociales en el trabajo en profesionales académicos argentinos, el cual ha sido aprobado por un Comité Ético/Científico de la Facultad de Psicología de la Universidad Nacional de Mar del Plata (código 15/H244). Una vez que se obtuvo una autorización expresa por parte de las máximas autoridades académicas de la Universidad para realizar la investigación, se envió un cuestionario virtual, desarrollado a través de la herramienta Google Drive ${ }^{\circledR}$, a un total de 687 potenciales participantes. La encuesta se encontró precedida por una descripción del estudio y un formulario de consentimiento informado. Sólo se otorgó acceso a la encuesta en línea cuando el consentimiento informado fue aceptado. Las respuestas a la encuesta fueron anónimas y cualquier información personal que permita identificar a los participantes es estrictamente confidencial. Los datos reportados en este artículo fueron recolectados entre octubre de 2015 y febrero de 2016.

\section{Análisis}

Las propiedades psicométricas del Copsoq-Istas 21 fueron examinadas en términos de (a) su consistencia interna (confiabilidad), para lo que se calculó el Coeficiente Alfa de Cronbach de manera global y por sub-escala; (b) su estructura factorial, mediante el empleo de técnicas de análisis factorial confirmatorio; (c) su validez convergente, por medio del método de la varianza media extraída; y (d) su validez discriminante, mediante comparaciones sucesivas entre la varianza media extraída y la varianza compartida. El puntaje total para cada factor de riesgo fue calculado como la suma de los puntajes correspondientes a cada uno de sus ítems y luego recategorizado en una escala compuesta por tres niveles ( 1 = baja exposición al factor de riesgo psicosocial, 3 = elevada exposición al factor de riesgo psicosocial), habiendo empleado para ello los baremos publicados por Moncada et ál. (2004). Finalmente, para analizar la influencia de un conjunto de variables sociodemográficas y laborales de los participantes sobre su percepción de exposición a riesgos psicosociales se realizaron análisis de regresiones múltiples. 


\section{Resultados}

\section{Propiedades psicométricas del Copsoq-Istas 21}

La confiabilidad del Copsoq-Istas 21 fue analizada en términos de su consistencia interna, para lo cual se calculó el Coeficiente Alfa de Cronbach. De manera similar a lo reportado por Alvarado et ál. (2009), se obtuvieron niveles mayores de consistencia interna para la escala global que para las dimensiones individuales que componen el instrumento. En efecto, mientras que la escala global posee una elevada confiabilidad $(\alpha=0,90)$, se registraron coeficientes alfa bajos a moderados para las seis dimensiones que componen el instrumento, oscilando los mismos entre 0,57 y 0,85 . Al respecto, debe mencionarse que la obtención de niveles de confiabilidad elevados para la escala completa y moderados para sus sub-escalas podría ser un indicador de redundancia y no de una mejora real en el nivel de consistencia interna de la escala global (Tavakol y Dennick, 2011), al ser el Coeficiente Alfa de Cronbach sensible a la cantidad de ítems incorporados en su cálculo. De manera consistente con lo encontrado en estudios previos (Alvarado et ál., 2009; Alvarado et ál., 2012), la dimensión Doble Presencia fue la que registró menor nivel de consistencia interna.

La validez de constructo del Copsoq-Istas 21 fue examinada en términos de su estructura factorial, validez convergente y validez discriminante. En primer lugar, y con el propósito de contrastar empíricamente el modelo teórico propuesto por Moncada et ál. (2005) y estudiar la estructura factorial del instrumento, se realizó un análisis factorial confirmatorio en IBM SPSS AMOS ${ }^{\circledR}$ (versión 22), mediante el método de ecuaciones estructurales. De manera previa, se verificó el cumplimiento de los supuestos mínimos para la aplicación del modelo factorial, a través del cálculo de la Medida de Adecuación Muestral de Kaiser-Meyer-0lkin (KM0) y de la Prueba de la Esfericidad de Bartlett. La obtención de un estadístico $\mathrm{KMO}=0,83$, en conjunto con la observación de diferencias estadísticamente significativas en la prueba de la esfericidad de Bartlett $\left(\chi^{2}=3.492,74\right.$; $\mathrm{df}=703 ; \mathrm{p}<0,01)$, revelaron que el empleo de la técnica de análisis factorial resulta apropiado.

Para el análisis factorial confirmatorio se siguió un procedimiento análogo al empleado por Piccolo, Judge, Takahashi, Watanabe y Locke (2005) y se utilizó un modelo de desagregación parcial, que consiste en la creación de dos o más variables observadas agregadas para cada variable latente (Dabholkar, Thorpe y Rentz, 1996). Siguiendo este enfoque, en lugar de introducir los reactivos específicos del cuestionario como las 
variables observadas correspondientes a cada una de las seis variables latentes (modelo de desagregación total), se construyeron parcelas de ítems para cada variable latente (Bagozzi y Edwards, 1998). Este procedimiento suele ser el más apropiado frente a la presencia de muestras pequeñas o moderadas, puesto que tiende a mejorar los índices de ajuste del modelo al reducir la cantidad de parámetros a ser estimados (Rocha y Chelladurai, 2012). Si bien en la literatura es posible identificar diferentes metodologías para la construcción de las parcelas en los modelos de desagregación parcial, se siguieron las recomendaciones metodológicas de Rocha y Chelladurai (2012) de utilizar el análisis factorial exploratorio (EFA) dentro de cada sub-escala para determinar los ítems específicos que integrarían cada parcela. ${ }^{1}$

En la evaluación del modelo se siguieron las recomendaciones de Bollen (1989) de reportar y comparar diversos índices de ajuste, tales como: el estadístico chi-cuadrado $\left(\chi^{2}\right)$, el comparative fit index (CFI), el goodness of fit index (GFI), el root mean square error of approximation (RMSEA) y el parsimony goodness of fit index (PGFI). Siguiendo a Byrne (2001), valores de CFI y GFI iguales o superiores a 0,90, de RMSEA de hasta 0,08 y de PGFI en torno a 0,50 constituyen indicadores de buen ajuste. Los resultados del análisis factorial confirmatorio revelaron que el modelo teórico provee un ajuste aceptable a los datos $\left(\chi^{2}=113,70 ; g l=50 ; p<0,01 ; C F I=0,92 ; G F I=0,92 ; \operatorname{RMSEA}=0,09\right.$; PGFI $=0,50)$, ver figura 1 .

La validez de constructo del Copsoq-Istas 21 (1.5) también fue analizada en términos de su validez convergente, entendida por Martínez-García y Martínez-Caro (2009) como el grado en que las medidas correspondientes a un constructo observan correlaciones elevadas entre sí. En la tabla 1 se presentan las cargas factoriales estandarizadas de los 38 ítems del instrumento. Como puede apreciarse, los pesos factoriales oscilaron entre 0,30 y 0,74 para la sub-escala de Exigencias Psicológicas, entre 0,41 y 0,75 para la de Control sobre el Trabajo, entre 0,21 y 0,84 para la de Apoyo Social y Liderazgo, entre 0,29

1 Con sustento en los resultados del EFA, los seis ítems correspondientes a la dimensión Exigencias Psicológicas fueron agrupados en dos parcelas de tres ítems cada una, calculadas como el promedio de sus puntajes (parcela 1: ítems 1 a 3, parcela 2: ítems 4 a 6). Para el caso del Control sobre el Trabajo, se construyeron dos parcelas de seis y cuatro ítems cada una (parcela 1: ítems 1 a 6, parcela 2: ítems 7 a 10). Con respecto al Apoyo Social y Liderazgo, los resultados del EFA sugirieron la construcción de tres parcelas, con 3, 5 y 2 ítems cada una (parcela 1: ítems 1 a 3; parcela 2: ítems 4, 5, 6, 8 y 10; parcela 3: ítems 7 y 9). Para el caso de la Estima, la Doble Presencia y la Inseguridad sobre el Futuro (con cuatro ítems cada una), se crearon dos parcelas compuestas por dos ítems cada una (Estima, parcela 1: ítems 1 y 4; Estima, parcela 2: ítems 2 y 3; Doble Presencia, parcela 1: ítems 1 y 3; Doble Presencia, parcela 2: ítems 2 y 4; Inseguridad sobre el Futuro, parcela 1: ítems 1 y 4; Inseguridad sobre el Futuro, parcela 2: ítems 2 y 3 ). 
y 0,66 para la de Doble Presencia, entre 0,54 y 0,90 para la de Estima y entre 0,57 y 0,78 para la de Inseguridad sobre el Futuro. En un siguiente paso, se procedió a calcular las cargas factoriales estandarizadas elevadas al cuadrado para cada uno de los 38 ítems y luego la Varianza Media Extraída (VME) para cada uno de los seis factores latentes. Los resultados revelan que sólo para la sub-escala de Estima se obtuvieron valores de VME superiores a 0,50 (indicador de adecuada validez convergente, ver Hair et ál., 2010).

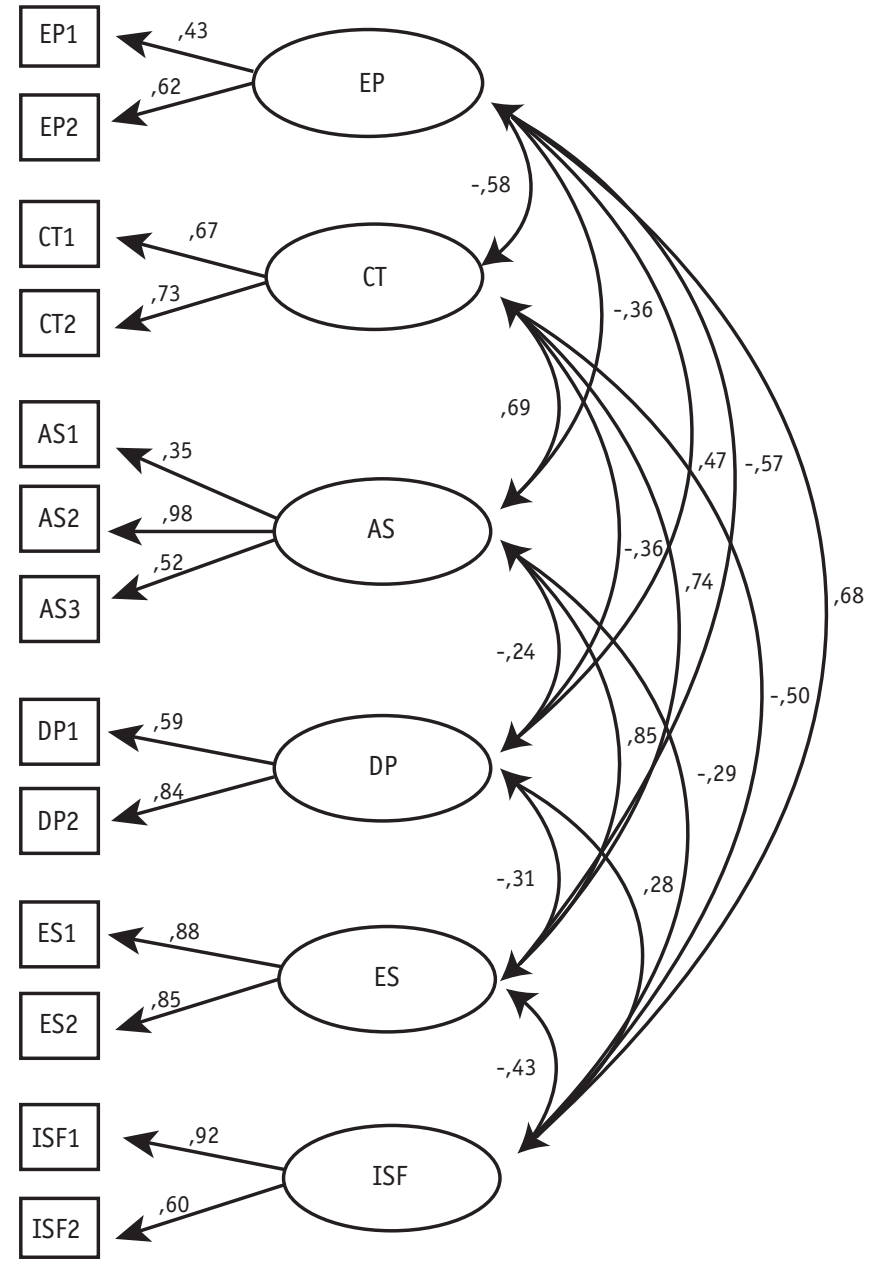

Figura 1. Resultados del análisis factorial confirmatorio

Nota: $\mathrm{n}=175, \mathrm{EP}=$ Exigencias Psicológicas, CT = Control sobre el Trabajo, DP = Doble Presencia, AS = Apoyo Social y Calidad del Liderazgo, ES = Estima, ISF = Inseguridad sobre el Futuro.

Fuente: elaboración propia. 


\section{Tabla 1}

Cargas factoriales, Varianza Media Extraída y niveles de confiabilidad

\begin{tabular}{|c|c|c|c|c|c|}
\hline Ítem & CFE & $\mathrm{CFE}^{2}$ & VME & Alfa & Alfa si se elimina ítem \\
\hline \multicolumn{6}{|c|}{ Exigencias psicológicas } \\
\hline EP\#1 & 0,41 & 0,17 & \multirow{6}{*}{0,26} & \multirow{6}{*}{0,66} & 0,62 \\
\hline EP\#2 & 0,36 & 0,13 & & & 0,61 \\
\hline EP\#3 & 0,30 & 0,09 & & & 0,64 \\
\hline EP\#4 & 0,65 & 0,42 & & & 0,61 \\
\hline EP\#5 & 0,74 & 0,55 & & & 0,58 \\
\hline EP\#6 & 0,44 & 0,19 & & & 0,66 \\
\hline \multicolumn{6}{|c|}{ Control sobre el trabajo } \\
\hline CT\#1 & 0,60 & 0,36 & \multirow{10}{*}{0,35} & \multirow{10}{*}{0,83} & 0,82 \\
\hline CT\#2 & 0,75 & 0,56 & & & 0,80 \\
\hline $\mathrm{CT} \# 3$ & 0,68 & 0,46 & & & 0,81 \\
\hline CT\#4 & 0,51 & 0,26 & & & 0,82 \\
\hline CT\#5 & 0,45 & 0,20 & & & 0,83 \\
\hline CT\#6 & 0,65 & 0,42 & & & 0,81 \\
\hline CT\#7 & 0,56 & 0,31 & & & 0,82 \\
\hline CT\#8 & 0,41 & 0,17 & & & 0,83 \\
\hline СТ\#9 & 0,62 & 0,38 & & & 0,81 \\
\hline CT\#10 & 0,59 & 0,35 & & & 0,82 \\
\hline \multicolumn{6}{|c|}{ Apoyo social y calidad del liderazgo } \\
\hline AS\#1 & 0,37 & 0,14 & \multirow{10}{*}{0,39} & \multirow{10}{*}{0,82} & 0,81 \\
\hline AS\#2 & 0,26 & 0,07 & & & 0,82 \\
\hline AS\#3 & 0,39 & 0,15 & & & 0,81 \\
\hline AS\#4 & 0,83 & 0,69 & & & 0,79 \\
\hline AS\#5 & 0,84 & 0,71 & & & 0,79 \\
\hline AS\#6 & 0,83 & 0,69 & & & 0,78 \\
\hline AS\#7 & 0,21 & 0,04 & & & 0,87 \\
\hline AS\#8 & 0,71 & 0,50 & & & 0,79 \\
\hline AS\#9 & 0,68 & 0,46 & & & 0,79 \\
\hline AS\#10 & 0,67 & 0,45 & & & 0,79 \\
\hline \multicolumn{6}{|c|}{ Doble presencia } \\
\hline DP\#1 & 0,62 & 0,38 & \multirow{4}{*}{0,26} & \multirow{4}{*}{0,57} & 0,47 \\
\hline $\mathrm{DP \# 2}$ & 0,66 & 0,44 & & & 0,50 \\
\hline DP\#3 & 0,29 & 0,08 & & & 0,54 \\
\hline DP\#4 & 0,38 & 0,14 & & & 0,47 \\
\hline
\end{tabular}




\begin{tabular}{cccccc}
\hline Ítem & CFE & CFE $^{2}$ & VME & Alfa & Alfa si se elimina ítem \\
\hline \multicolumn{5}{c}{ Estima } \\
ES\#1 & 0,87 & 0,76 & & \\
ES\#2 & 0,90 & 0,81 & 0,61 & 0,85 & 0,78 \\
ES\#3 & 0,54 & 0,29 & & & 0,77 \\
ES\#4 & 0,76 & 0,58 & 0,88 \\
\hline \multicolumn{5}{c}{ Inseguridad sobre el futuro } \\
ISF\#1 & 0,57 & 0,32 & \\
ISF\#2 & 0,77 & 0,59 & 0,80 \\
ISF\#3 & 0,78 & 0,61 & 0,46 & 0,76 \\
ISF\#4 & 0,57 & 0,32 & & 0,68 \\
\hline
\end{tabular}

Nota: $\mathrm{CF}=$ carga factorial, $\mathrm{VME}=$ varianza media extraída.

Fuente: elaboración propia.

La validez discriminante, representativa del grado en que los seis factores latentes del Copsoq-Istas no comparten una varianza sustancial entre sí, fue analizada siguiendo el procedimiento recomendado por Fornell y Larcker (1981). Para estos autores, existe validez discriminante entre dos variables latentes si la varianza compartida entre ellas (ver las 15 combinaciones listadas en la tabla 2) resulta menor que la varianza media extraída. La varianza compartida entre los factores osciló entre 0,05 y 0,52, con un promedio de 0,15 . Los resultados reportados en la tabla 2 revelan niveles satisfactorios de validez discriminante para cinco de las seis dimensiones teóricas que componen el instrumento. Sólo para el caso de las dimensiones Apoyo Social y Liderazgo y Estima se observaron niveles poco satisfactorios de validez discriminante, al haberse obtenido valores de varianza compartida $(0,52)$ mayores a los de la varianza media extraída $(0,50)$.

\section{Tabla 2}

Resultados del análisis de validez discriminante

\begin{tabular}{lcc}
\hline \multicolumn{1}{c}{ Combinaciones } & VME & VC \\
\hline Exigencias Psicológicas - Control sobre el Trabajo & 0,31 & 0,09 \\
Exigencias Psicológicas - Apoyo Social & 0,33 & 0,05 \\
Exigencias Psicológicas - Doble Presencia & 0,26 & 0,08 \\
Exigencias Psicológicas - Estima & 0,44 & 0,12 \\
Exigencias Psicológicas - Inseguridad sobre el Futuro & 0,36 & 0,15 \\
Control sobre el Trabajo - Apoyo Social & 0,37 & 0,34 \\
Control sobre el Trabajo - Doble Presencia & 0,31 & 0,07 \\
\hline & & Continúa $\rightarrow$
\end{tabular}




\begin{tabular}{lcc}
\hline \multicolumn{1}{c}{ Combinaciones } & VME & VC \\
\hline Control sobre el Trabajo - Estima & 0,48 & 0,30 \\
Control sobre el Trabajo - Inseguridad sobre el Futuro & 0,41 & 0,13 \\
Apoyo Social - Doble Presencia & 0,33 & 0,05 \\
Apoyo Social - Estima & 0,50 & 0,52 \\
Apoyo Social - Inseguridad sobre el Futuro & 0,43 & 0,11 \\
Doble Presencia - Estima & 0,44 & 0,05 \\
Doble Presencia - Inseguridad sobre el Futuro & 0,36 & 0,05 \\
Estima - Inseguridad sobre el Futuro & 0,54 & 0,13 \\
\hline
\end{tabular}

Nota: $\mathrm{VME}=$ varianza media extraída, $\mathrm{CV}$ = varianza compartida.

Fuente: elaboración propia.

\section{Nivel de exposición a factores psicosociales de riesgo}

En la tabla 3 se presentan las medias, desviaciones estándar y correlaciones para cada una de las seis dimensiones que componen el modelo Copsoq-Istas 21. Por el lado de las demandas laborales, se observa que el factor que genera mayor nivel de exposición psicosocial en los docentes participantes corresponde a la Doble Presencia $(M=2,12$; $\mathrm{DE}=0,71)$. Focalizando ahora la atención sobre los recursos laborales, se aprecia que el factor que genera mayor nivel de exposición está dado por la Estima en niveles insuficientes $(M=2,66 ; D E=0,85)$.

\section{Tabla 3}

Estadísticos descriptivos y correlaciones por dimensión

\begin{tabular}{|c|c|c|c|c|c|c|c|c|c|c|}
\hline Escala & M & $\mathrm{DE}$ & $M_{s}$ & $\mathrm{DE}_{\mathrm{s}}$ & 1 & 2 & 3 & 4 & 5 & 6 \\
\hline 1. Exigencias psicológicas & 1,92 & 0,55 & 11,53 & 3,29 & 1 & & & & & \\
\hline 2. Control sobre el trabajo & 3,04 & 0,58 & 30,39 & 5,84 & $-0,30$ & 1 & & & & \\
\hline 3. Apoyo social y liderazgo & 2,83 & 0,65 & 28,32 & 6,51 & $-0,23$ & 0,58 & 1 & & & \\
\hline 4. Doble presencia & 2,12 & 0,71 & 8,47 & 2,85 & 0,29 & $-0,27$ & $-0,24$ & 1 & & \\
\hline 5. Estima & 2,66 & 0,85 & 10,63 & 3,40 & $-0,34$ & 0,55 & 0,72 & $-0,23$ & 1 & \\
\hline 6. Inseguridad sobre el futuro & 1,31 & 0,89 & 5,26 & 3,57 & 0,38 & $-0,35$ & $-0,33$ & 0,22 & $-0,36$ & 1 \\
\hline
\end{tabular}

Nota: $\mathrm{n}=175, \mathrm{M}=$ Media, $\mathrm{DE}=$ Desviación estándar, $\mathrm{M}_{\mathrm{s}}=$ Media de la suma, $\mathrm{DE}_{\mathrm{s}}=$ Desviación estándar de la suma. La totalidad de las correlaciones reportadas resultan significativas al nivel de $p<0,01$.

Fuente: elaboración propia.

Empleando los baremos publicados por Moncada et ál. (2004), las sumas de los puntajes de las seis dimensiones del modelo fueron recategorizadas en una escala de tres 
niveles, indicativa del nivel de prevalencia de cada factor de riesgo psicosocial. Como puede observarse en la tabla 4, la mayor parte de los participantes reportan niveles de exposición bajos a moderados para las dimensiones Apoyo Social y Liderazgo, Control sobre el Trabajo e Inseguridad sobre el Futuro. Sin embargo, nótese que poco más del $90 \%$ de los docentes reportan niveles moderados a elevados de exposición a Exigencias Psicológicas y cerca del 34\% manifiestan recibir niveles insuficientes de Estima. Nuevamente, la Doble Presencia representa la dimensión del trabajo para la que se registró mayor nivel de riesgo psicosocial, observándose que un $76 \%$ de los docentes reportó una exposición desfavorable a dicho factor.

\section{Tabla 4}

Niveles de exposición para cada factor de riesgo

\begin{tabular}{lccc}
\hline \multicolumn{1}{c}{ Dimensión } & \multicolumn{3}{c}{ Nivel de exposición (en \%) } \\
\cline { 2 - 4 } & Favorable & Intermedia & Desfavorable \\
\hline 1. Exigencias psicológicas & 9,14 & 45,14 & 45,71 \\
2. Control sobre el trabajo & 82,29 & 13,71 & 4,00 \\
3. Apoyo social y liderazgo & 33,71 & 40,00 & 26,29 \\
4. Doble presencia & 2,29 & 21,71 & 76,00 \\
5. Estima & 32,00 & 34,29 & 33,71 \\
6. Inseguridad sobre el futuro & 44,57 & 45,71 & 9,71 \\
\hline
\end{tabular}

Nota: $\mathrm{n}=175$.

Fuente: elaboración propia.

\section{Influencia de factores sociodemográficos y laborales en la exposición a Riesgos Psicosociales}

Con el propósito de analizar la influencia ceteris paribus de un conjunto de variables sociodemográficas y otras relativas a las condiciones de empleo sobre la percepción de exposición de los docentes a los seis factores de riesgo psicosocial sugeridos por Moncada et ál. (2005), se construyeron modelos de regresiones múltiples a través del método de mínimos cuadrados ordinarios (OLS). ${ }^{2}$ Siendo la variable respuesta la exposición

2 Previamente se verificó que los datos dieran cumplimiento a los supuestos mínimos para la aplicación del análisis de regresión múltiple: (a) normalidad, mediante la conducción de la prueba de Shapiro-Wilk; (b) no colinealidad perfecta, mediante el cálculo del Factor de Inflación de Varianza (VIF), resultando aceptables valores inferiores a 10; (c) homocedasticidad, mediante la conducción de la prueba de Breusch-Pagan. En todos los casos los resultados fueron satisfactorios. 
percibida a cada uno de los seis factores de riesgo psicosocial, se introdujeron estos cinco predictores, género: variable dummy donde el género femenino fue considerado el grupo base, edad: variable cuantitativa continua, dedicación: variable dummy donde la dedicación tiempo parcial fue considerada el grupo base, cargo: variable dummy donde el cargo de Auxiliar fue considerado el grupo base, formación académica: variable dummy donde la formación de pregrado fue considerada el grupo base.

Los resultados (ver tabla 5) revelan que la percepción de exposición a Exigencias Psicológicas tiende a reducirse a mayor edad $(\beta=-0,0128 ; p<0,10)$, sugiriendo que los docentes más jóvenes constituyen el grupo social más vulnerable a este factor. Más aún, aquellos docentes con título de Doctor parecieran experimentar exigencias psicológicas un $8 \%$ superiores que aquellos con titulación de pregrado $(\beta=0,2747 ; p<0,05)$. En cuanto al Control sobre el Trabajo, se obtuvo que sólo el cargo del docente logra explicar de manera significativa la percepción de exposición a este factor de riesgo psicosocial. En efecto, y de manera consistente con lo esperado, aquellos docentes con un cargo de Profesor parecieran percibir mayor control sobre su trabajo que los Auxiliares (alrededor de un $5 \%$ mayor; $\beta=0,2506 ; p<0,05)$, ceteris paribus el resto de las variables explicativas consideradas. En relación con la Inseguridad sobre el Futuro, los resultados del análisis de regresiones múltiples revelan que los Profesores parecieran percibir menor exposición a este factor que los Auxiliares (alrededor de un 9\% menor; $\beta=-0,4472 ; p<0,01$ ). Finalmente, con respecto a la Doble Presencia, se obtuvo que el género del docente y la formación académica explican de manera significativa la percepción de exposición a este factor. En cuanto al género, se observa que los hombres parecieran experimentar alrededor de un $9 \%$ menos de doble presencia que las mujeres $(\beta=-0,4369 ; p<0,01)$, ceteris paribus. Además, mayores niveles de doble presencia son reportados por los Doctores (alrededor de un $11 \%$ mayores; $\beta=0,5528 ; p<0,01$ ), en comparación con aquellos docentes que únicamente poseen titulación de pregrado.

\section{Tabla 5}

Análisis de regresiones múltiples

\begin{tabular}{lcccccc}
\hline \multicolumn{1}{c}{ Variable } & $\begin{array}{c}\text { Exigencias } \\
\text { psicológicas }\end{array}$ & $\begin{array}{c}\text { Control sobre el } \\
\text { trabajo }\end{array}$ & $\begin{array}{c}\text { Inseguridad } \\
\text { frente al futuro }\end{array}$ & $\begin{array}{c}\text { Apoyo Socialy } \\
\text { Liderazgo }\end{array}$ & Doble presencia & Estima \\
\hline Género & 0,0040 & $-0,0317$ & 0,1696 & $-0,0668$ & $-0,4369 * * *$ & 0,0647 \\
masculino & $(0,0925)$ & $(0,0988)$ & $(0,1494)$ & $(0,1135)$ & $(0,1115)$ & $(0,1472)$ \\
\hline \multirow{2}{*}{ Edad } & $-0,0128^{*}$ & $-0,0044$ & $-0,0086$ & 0,0126 & $-0,0126$ & 0,0056 \\
& $(0,0067)$ & $(0,0072)$ & $(0,0108)$ & $(0,0082)$ & $(0,0081)$ & $(0,0107)$ \\
\hline
\end{tabular}




\begin{tabular}{lcccccc}
\hline \multicolumn{1}{c}{ Variable } & $\begin{array}{c}\text { Exigencias } \\
\text { psicológicas }\end{array}$ & $\begin{array}{c}\text { Control sobre el } \\
\text { trabajo }\end{array}$ & $\begin{array}{c}\text { Inseguridad } \\
\text { frente al futuro }\end{array}$ & $\begin{array}{c}\text { Apoyo Socialy } \\
\text { Liderazgo }\end{array}$ & Doble presencia & Estima \\
\hline $\begin{array}{l}\text { Dedicación full- } \\
\text { time }\end{array}$ & 0,0376 & 0,0328 & $-0,2561$ & 0,0467 & 0,0488 & $-0,1380$ \\
\hline $\begin{array}{l}\text { Cargo de } \\
\text { Profesor }\end{array}$ & $-0,0924$ & $0,2506^{* *}$ & $-0,4472 * * *$ & 0,0497 & 0,0629 & 0,2410 \\
\hline \multirow{2}{*}{ Antigüedad } & $(0,1059)$ & $(0,1131)$ & $(0,1710)$ & $(0,1299)$ & $(0,1277)$ & $(0,1684)$ \\
\hline Título de & 0,0054 & 0,0047 & 0,0112 & $-0,0061$ & $-0,0102$ & $-0,0145$ \\
Máster & $(0,0078)$ & $(0,0083)$ & $(0,0126)$ & $(0,0096)$ & $(0,0094)$ & $(0,0124)$ \\
\hline Título de & 0,0042 & 0,1597 & 0,1663 & $-0,0166$ & 0,1387 & 0,0885 \\
Doctor & $(0,1132$ & $(0,1208)$ & $(0,1828)$ & $(0,1389)$ & $(0,1364)$ & $(0,1800)$ \\
\hline \multirow{2}{*}{ Intercepto } & $0,2747 * *$ & 0,1650 & 0,3527 & $-0,0301$ & $0,5528 * * *$ & 0,0516 \\
& $(0,1377)$ & $(0,1470)$ & $(0,2224)$ & $(0,1689)$ & $(0,1660)$ & $(0,2116)$ \\
\hline $\mathrm{R}^{2}$ & $(0,2132)$ & $(0,2275)$ & $(0,3442)$ & $(0,2615)$ & $(0,2569)$ & $(0,3390)$ \\
\hline
\end{tabular}

Nota: $n=175 .{ }^{*} p<0,10 ;{ }^{* *} p<0,05 ;{ }^{* *} p<0.01$. Errores estándar entre paréntesis.

Fuente: elaboración propia.

\section{Discusión y conclusiones}

La docencia universitaria se está convirtiendo poco a poco en una ocupación de alto riesgo psicosocial, constituyendo en la actualidad una de las más estresantes (Fredman y Doughney, 2012; Houtman, 2005; Jordan et ál., 2003; Kinman y Wray, 2013; Salanova et ál., 2005). En efecto, el ejercicio de la profesión académica le exige al docente universitario niveles elevados de sobrecarga mental, al requerirle elevada concentración, precisión y atención a los detalles; y emocional, al implicarle su interacción con diferentes actores, tales como estudiantes, directivos, pares y subordinados. Pero, además, los riesgos psicosociales emergentes de la exposición elevada a estas demandas se ven agudizados cuando los niveles de ciertos recursos laborales que son fundamentales para el logro de las metas del trabajo y, al mismo tiempo, esenciales para el crecimiento y el desarrollo personal, tales como el reconocimiento, el apoyo social, el liderazgo y las compensaciones materiales, resultan insuficientes (Kinman y Wray, 2013; Fredman y Doughney, 2012).

El primer aporte de esta investigación consistió en el estudio de las propiedades psicométricas del Cuestionario para la Evaluación de Riesgos Psicosociales en el Trabajo Copsoq-Istas 21 , en su versión 1.5, en una muestra de docentes universitarios argentinos. 
De manera similar a lo reportado en investigaciones previas, los resultados de este estudio revelaron que el instrumento presenta una adecuada consistencia interna, dada por la obtención de coeficientes alfa moderados a elevados tanto para la escala completa como para las sub-escalas que la componen. Las sub-escalas de Exigencias Psicológicas y de Doble Presencia constituyeron las dos excepciones a la proposición anterior. En efecto, y si bien la primera observó un coeficiente alfa cercano a lo que podría ser considerado un nivel apropiado (Nunnally y Bernstein, 1994), la dimensión Doble Presencia arrojó un coeficiente demasiado bajo como para ser considerado aceptable. Puesto que este resultado ya ha sido reportado en el pasado (Alvarado et ál., 2009; Alvarado et ál., 2012), la evidencia anterior podría ser suficiente para poner en cuestionamiento la operacionalización de esta sub-dimensión. Se considera necesario que futuros estudios introduzcan modificaciones en los reactivos correspondientes a la sub-escala de Doble Presencia con el propósito de incrementar su consistencia interna, lo que contribuiría, a su vez, a mejorar las propiedades psicométricas del instrumento.

La validación de constructo del Copsoq-Istas 21 (versión 1.5) también resultó ligeramente problemática. En efecto, y si bien los resultados del análisis factorial confirmatorio brindaron un soporte aceptable a una estructura compuesta por seis dimensiones latentes, al mismo tiempo, se observaron niveles poco satisfactorios de validez convergente para todas ellas, con excepción de la dimensión Estima. Una forma de mejorar la validez convergente del instrumento podría ser eliminar aquellos ítems que observaron bajos pesos factoriales dentro de su correspondiente dimensión teórica. Otra alternativa complementaria podría ser separar aquellas dimensiones problemáticas en sub-dimensiones, empleando para ello los resultados de un análisis factorial exploratorio. Con respecto a la validez discriminante, los análisis practicados revelaron que la varianza compartida entre la sub-escala de Apoyo Social y Liderazgo y la de Estima resulta superior a su varianza media extraída, indicando niveles insatisfactorios de validez divergente. Futuras investigaciones podrían considerar agrupar ambas dimensiones dentro de una dimensión agregada de mayor amplitud.

El segundo aporte de este artículo consistió en la medición y análisis de la prevalencia de seis categorías genéricas de riesgos psicosociales en el trabajo en una muestra de docentes universitarios argentinos. Los resultados de esta investigación revelaron que los participantes se hallan expuestos a niveles elevados de Doble Presencia e insuficiente Estima. En sintonía con el enfoque adoptado por García et ál. (2016), este estudio también facilitó la identificación de los grupos de docentes más vulnerables (aquellos que reportaron una exposición significativamente mayor a las distintas categorías de 
riesgos psicosociales) a través de un análisis de la influencia ceteris paribus de un conjunto de variables demográficas (género, edad y nivel educativo) y laborales (antigüedad, jerarquía del cargo y dedicación horaria) sobre la percepción de exposición de los participantes a los distintos factores de riesgo.

Con respecto al género, se observó que el mismo influye de manera significativa en el nivel de exposición a Doble Presencia. En efecto, y de manera consistente con lo reportado en estudios previos, la prevalencia de Doble Presencia resultó ser mayor en las mujeres que en los hombres, significando esto último que las primeras parecieran experimentar mayores dificultades para balancear las exigencias de la vida académica y las provenientes del ámbito familiar o doméstico (García et ál., 2016; Unda et ál., 2016; Bobbitt-Zeher, 2011; Rojas y Rodríguez, 2011; Shockley y Allen, 2007).

Además, se observó que la edad incide de manera significativa sobre la percepción de exposición a Exigencias Psicológicas. De manera similar a lo reportado por García et ál. (2016), de este estudio surgió que el grupo más vulnerable pareciera estar compuesto por aquellos docentes menores de 40 años. Al menos dos mecanismos podrían contribuir a explicar estas diferencias. Por un lado, es posible que los docentes más jóvenes posean realmente condiciones laborales menos favorables, por ejemplo, en términos de estabilidad laboral, status y carga de trabajo. Por el otro, también es posible que aquellos docentes con menor experiencia en el ejercicio de la profesión posean menores recursos personales ( $p$. ej. autoeficacia) para hacer frente a las demandas del trabajo y que, en consecuencia, las perciban y experimenten como más estresantes (Pujol-Cols y Dabos, 2018).

En conexión con lo enunciado en el párrafo precedente, los resultados de este estudio revelaron que la jerarquía del cargo ocupado incide de manera significativa sobre la percepción de Control sobre el Trabajo y de Inseguridad sobre el Futuro, habiéndose identificado que el riesgo pareciera ser mayor en Auxiliares que en Profesores. Con respecto a la primera dimensión, los niveles menores de Control sobre el Trabajo detectados en los Auxiliares resultan razonables en virtud de su menor capacidad para influir de manera directa sobre la planificación de las asignaturas y la definición de líneas de investigación, viéndose obligados a trabajar en un marco de constreñimientos definidos previamente por los Profesores. Con respecto a la segunda dimensión, se observó que los Auxiliares parecieran experimentar mayor inseguridad laboral que los Profesores, probablemente en virtud de su menor nivel de consolidación en el sistema universitario y, en particular, en la universidad de su afiliación. 


\section{Limitaciones}

Una primera limitación de este estudio reside en su carácter descriptivo. Futuras investigaciones deberían abocarse al análisis de los efectos de la exposición a riesgos psicosociales (tanto de manera general como por factor de riesgo) sobre otros criterios de relevancia para la Psicología Organizacional, como lo son la satisfacción laboral, la ciudadanía organizacional, el compromiso organizacional y el work engagement. Además, y en línea con el notable crecimiento experimentado por el disposicionalismo dentro de la Psicología, podría ser interesante indagar en el rol que asumen los rasgos de la personalidad de los individuos (p. ej. la autoeficacia) en su percepción de exposición a riesgos psicosociales en el trabajo (ver Pujol-Cols y Dabos, 2018 para una revisión). Por otro lado, y con el propósito de evitar análisis excesivamente reduccionistas, se coincide con Heller, Ferris, Brown y Watson (2009) en que la Psicología Organizacional requiere continuar explorando los mecanismos y constructos psicológicos que ejercen papeles mediadores o moderadores en esta dinámica de relaciones.

En segundo lugar, y de manera compatible con la metodología dominante en Psicología Organizacional, este estudio se sustentó en el uso de escalas de auto-informe. Las limitaciones enfrentadas por esta metodología ya han sido observadas previamente en la literatura (ver Krumpal, 2013). En efecto, y puesto que existe en los individuos una tendencia a presentarse a sí mismos de la manera más favorable posible, fenómeno llamado sesgo de conformidad social, algunos participantes de este estudio podrían haber sido incapaces o no haber estado dispuestos a responder verazmente preguntas asociadas con temáticas sensibles (Piedmont, McCrae y Riemann, 2000), como lo son las laborales. Como resultado, futuras investigaciones deberían abogar por triangular la evidencia recolectada a través de los cuestionarios de auto-informe con la obtenida por vía de otras estrategias, como las entrevistas en profundidad y la observación.

Como tercera limitación, se señala que este estudio se sustentó en datos de corte transversal, lo que impide a los autores postular relaciones de causa-efecto. En su lugar, la conducción de investigaciones longitudinales permitiría comprender más profundamente los procesos y mecanismos por los que los riesgos psicosociales en el trabajo afectan cognitiva, afectiva y conductualmente a los individuos y grupos en contextos organizacionales. Más aún, el empleo de este tipo de diseños metodológicos permitiría robustecer los modelos sugeridos hasta el momento en la literatura, al facilitar el control de los efectos del transcurso del tiempo sobre el comportamiento de las variables bajo estudio. 
Finalmente, esta investigación implicó la medición y análisis de categorías genéricas de riesgos psicosociales a las que se encuentran expuestos los docentes universitarios argentinos. Sin embargo, se coincide con García et ál. (2016) en que el empleo exclusivo de taxonomías genéricas conduce a ignorar problemáticas específicas que afectan a ciertos contextos organizacionales o profesiones, por lo que se sugiere que futuras investigaciones realicen una adaptación de los instrumentos genéricos dominantes a las particularidades de la profesión académica en Argentina. A modo de ejemplo, la literatura previa ha reconocido que la alta proporción de contratos de tiempo parcial que suelen caracterizar a las universidades públicas argentinas (alrededor del 75\% del total de dedicaciones; ver García de Fanelli y Moguillansky, 2014) tiende a traducirse en una mayoría de docentes que no ejercen la profesión académica como actividad laboral principal. Dado que la versión 1.5 del Copsoq-Istas 21 reduce la Doble Presencia exclusivamente a la dificultad para responder simultáneamente a las demandas del empleo y las del ámbito doméstico o familiar, la complejidad de la situación ocupacional antes descripta habilita a pensar que podrían existir otras demandas adicionales a las de la vida familiar que pueden exponer a los docentes de tiempo parcial a riesgo psicosocial como, por ejemplo, las que emergen de la dificultad para compatibilizar la actividad de enseñanza con las demandas provenientes del ejercicio de la profesión liberal. Elementos como los anteriores deberían ser contemplados en futuras investigaciones que impliquen el estudio de los riesgos psicosociales en docentes universitarios argentinos.

\section{Implicancias para la práctica}

El objetivo de una gestión de personal exitosa no consiste solamente en dirigir los esfuerzos del talento humano hacia el logro efectivo de los objetivos organizacionales, sino también en diseñar puestos, métodos y ambientes de trabajo saludables que estimulen el desarrollo de las personas en las organizaciones. Desde esta concepción, y en virtud de las profundas transformaciones que se han suscitado en el sistema universitario en las últimas décadas y, como resultado, en la profesión académica, se sostiene que resulta fundamental que las universidades diseñen estrategias y políticas que contemplen los riesgos psicosociales que afectan a sus académicos no sólo para lograr su retención y desarrollo sino, también, para preservar su salud física y mental. Para que este proceso resulte efectivo, se cree esencial que los Estados, por su rol en la sanción y control de las leyes, y las organizaciones sindicales, por su rol en la elaboración y negociación de los Convenios Colectivos de Trabajo, asuman un papel activo orientando el comportamiento de las universidades hacia prácticas laborales socialmente responsables que contemplen la protección de la salud psicosocial de sus colaboradores. 
Quienes se ocupan de la gestión de las universidades requieren comprender que una exitosa adecuación entre el empleado y la organización constituye una responsabilidad de ambos actores. En efecto, y puesto que una adaptación exclusiva por parte del empleado al contexto organizacional resulta poco probable dada la naturaleza relativamente estable de la personalidad (ver Dormann, Fay, Zapfy Frese, 2006; Caspi, Roberts y Shiner, 2005), se coincide con Moncada y Llorens (2004) en que resulta esencial que las universidades introduzcan cambios en la organización del trabajo, que propendan a reducir las demandas laborales y/o a incrementar los recursos laborales con el propósito de mitigar su impacto psicosocial sobre los docentes, en particular en aquellos aspectos ligados a la Doble Presencia, las Exigencias Psicológicas y la Estima.

Finalmente, se sostiene que un examen psicométrico minucioso de las diferentes versiones del Copsoq-Istas 21 en diversidad de muestras profesionales y contextos laborales resulta fundamental si se pretende desarrollar una versión económica, pero igualmente válida y confiable, que pueda ser utilizada de manera más efectiva en la investigación científica. Esto resulta particularmente esencial para aquellos diseños de investigación que pretendan explorar la vinculación de los riesgos psicosociales con otros constructos de relevancia para la Psicología Organizacionaly, específicamente, la Salud Ocupacional, como, por ejemplo, la satisfacción laboral, el work engagement, la ciudadanía organizacional, el compromiso organizacional o el burnout.

\section{Referencias}

Acevedo, G., Sánchez, J., Farías, M. y Fernández, A. (2013). Riesgos psicosociales en el equipo de salud de hospitales públicos de la Provincia de Córdoba, Argentina. Ciencia y Trabajo, 15(48), 140-147.

Alvarado, R., Marchetti, N., Villalón, M. et ál. (2009). Adaptación y análisis psicométrico de un cuestionario para evaluar riesgos psicosociales en el trabajo en Chile: versión media del CoPsoQ. Revista Chilena de Salud Pública, 13(1), 7-16.

Alvarado, R., Pérez-Franco, J., Saavedra, N. et ál. (2012). Validación de un cuestionario para evaluar riesgos psicosociales en el ambiente laboral en Chile. Revista Médica de Chile, 140(9), 1154-1163.

Avey, J., Reichard, R., Luthans, F. \& Mhatre, K. (2011). Meta-analysis of the impact of positive psychological capital on employee attitudes, behaviors, and performance. Human Resource Development Quarterly, 22(2), 127-152.

Bagozzi, R. \& Edwards, J. (1998). A general approach for representing constructs in organizational research. Organizational Research Methods, 1(1), 45-87. 
Bakker, A. \& Demerouti, E. (2014). Job demands-resources theory. In C. Cooper and P. Chen (eds.), Wellbeing. A Complete reference guide (pp. 37-64). Chichester: Wiley-Blackwell. Blanch, J., Sahagún, M. y Cervantes, G. (2010). Estructura factorial del cuestionario de condiciones de trabajo. Revista de Psicología del Trabajo y de las Organizaciones, 26(3), 175-189.

Blaschke, S., Frost, J. \& Hattke, F. (2014). Towards a micro foundation of leadership, governance, and management in universities. Higher Education, 68(5), 711-732.

Bobbitt-Zeher, D. (2011). Gender discrimination at work: Connecting gender stereotypes, institutional policies, and gender composition of workplace. Gender \& Society, 25(6), 764-786.

Bollen, K. (1989). A new incremental fit index for general structural equation models. Sociological Methods and Research, 17(3), 303-316.

Botero, C. (2012). Riesgo psicosocial intralaboral y "burnout" en docentes universitarios de algunos países latinoamericanos. Cuadernos de Administración (Universidad del Valle), 28(48), 117-132.

Bradshaw, P. \& Fredette, C. (2009). Academic governance of universities: Reflections of a senate chair on moving from theory to practice and back. Journal of Management Inquiry, 18(2), 123-133.

Byrne, B. (2001). Structural equation modeling: Perspectives on the present and the future. International Journal of Testing, 1(3-4), 327-334.

Caspi, A., Roberts, B. \& Shiner, R. (2005). Personality development: Stability and change. Annual Review of Psychology, 56(1), 453-484.

Charria, V., Sarsosa, K. y Arenas, F. (2011). Factores de riesgo psicosocial laboral: métodos e instrumentos de evaluación. Revista Facultad Nacional de Salud Pública, 29(4), 379-391.

Dabholkar, P., Thorpe, D. \& Rentz, J. (1996). A measure of service quality for retail stores: scale development and validation. Journal of the Academy of Marketing Science, 24(1), 3-16.

Demerouti, E., Bakker, A., Nachreiner, F. \& Schaufeli, W. (2001). The job demands-resources model of burnout. Journal of Applied Psychology, 86(3), 499-512.

Dormann, C., Fay, D., Zapf, D. \& Frese, M. (2006). A state-trait analysis of job satisfaction: On the effect of core self-evaluations. Applied Psychology, 55(1), 27-51.

Fornell, C. \& Larcker, D. (1981). Evaluating structural equation models with unobservable variables and measurement error. Journal of Marketing Research, 18(1), 39-50.

Fredman, N. \& Doughney, J. (2012). Academic dissatisfaction, managerial change and neo-liberalism. Higher Education, 64(1), 41-58.

García de Fanelli, A. y Moguillansky, M. (2014). La docencia universitaria en Argentina Obstáculos en la carrera académica. Education Policy Analysis Archives, 22, 1-18.

García, M., Iglesias, S., Saleta, M. \& Romay, J. (2016). Riesgos psicosociales en el profesorado de enseñanza universitaria: diagnóstico y prevención. Revista de Psicología del Trabajo y de las Organizaciones, 32(3), 173-182. 
Hair, J., Black, W., Babin, B. \& Anderson, R. (2010). Multivariate data analysis: A global perspective. New Jersey: Pearson Prentice Hall.

Hakanen, J., Bakker, A. \& Schaufeli, W. (2006). Burnout and work engagement among teachers. Journal of School Psychology, 43(6), 495-513.

Heller, D., Ferris, D., Brown, D. \& Watson, D. (2009). The influence of work personality on job satisfaction: Incremental validity and mediation effects. Journal of Personality, 77(4), 1051-1084.

Houtman, I. (2005). Work-related stress. Dublin: European Foundation for the Improvement of Living and Working Conditions.

Instituto Navarro de Salud Laboral (2002). Factores psicosociales, identificación de situaciones de riesgo. Navarra: Fondo de Publicaciones del Gobierno de Navarra.

Jordan, J., Gurr, E., Tinline, G., Giga, S. et ál. (2003). Beacons of excellence in stress prevention. Manchester: Robertson Cooper and UMIST.

Karasek Jr., R. (1979). Job demands, job decision latitude, and mental strain: Implications for job redesign. Administrative Science Quarterly, 24(2), 285-308.

Karasek, R., Brisson, C., Kawakami, N., Houtman, I., Bongers, P. \& Amick, B. (1998). The Job Content Questionnaire (JCQ): An instrument for internationally comparative assessments of psychosocial job characteristics. Journal of Occupational Health Psychology, 3(4), 322-355.

Kinman, G. \& Wray, S. (2013). Higher stress: A survey of stress and well-being among staffin higher education. UK: University and College Union.

Kristensen, T., Hannerz, H., Høgh, A. \& Borg, V. (2005). The Copenhagen Psychosocial Questionnaire-a tool for the assessment and improvement of the psychosocial work environment. Scandinavian Journal of Work, Environment y Health, 31(6), 438-449.

Krumpal, I. (2013). Determinants of social desirability bias in sensitive surveys: A literature review. Quality \& Quantity, 47(4), 2025-2047.

Llorens, S., Cifre, E., Salanova, M. \& Martínez, I. (2003a). Burnout in University Teachers: A gender differential study. In $11^{\text {th }}$ European Congress on Work and Organizational Psychology.

Llorens, S., García-Renedo, M., Cifre, E. \& Salanova, M. (2003b). Burnout and Engagement as antecedents of self-efficacy in Secondary teachers: A longitudinal study. In $11^{\text {th }}$ European Congress on work and Organizational Psychology.

Luthans, F. (2002). Positive organizational behavior: Developing and managing psychological strengths. The Academy of Management Executive, 16(1), 57-72.

Manassero, M., Vázquez, A., Ferrer, V., Fornés, J., Fernández, M. y Queimadelos, M. (2003). Estrés y burnout en la enseñanza. Palma de Mallorca: Ediciones UIB.

Martínez-García, J. y Martínez-Caro, L. (2009). La validez discriminante como criterio de evaluación de escalas. ¿Teoría o estadística? Universitas Psychologica, 8(1), 27-36. 
Meliá, J., Nogareda, C., Lahera, M., Duro, A., Peiró, J., Salanova, M. y Gracia, D. (2006). Principios comunes para la evaluación de los riesgos psicosociales en la empresa. En J. Meliá et ál. (eds.), Perspectivas de intervención en riesgos psicosociales. Evaluación de riesgos (pp. 13-36). Barcelona: Foment del Treball Nacional.

Ministerio de la Protección Social (2010). Batería de instrumentos para la evaluación de factores de riesgo psicosocial. Bogotá: Ministerio de la Protección Social.

Moncada, S. y Llorens, C. (2004). Evaluación y acción preventiva ante el riesgo psicosocial: El método ISTAS-21 (COPSOQ). Gestión Práctica de Riesgos Laborales, 5(1), 12-20.

Moncada, S., Llorens, C. y Kristensen, T. (2004). Método ISTAS 21 (CoPsoQ). Manual para la evaluación de riesgos psicosociales en el trabajo. Instituto Sindical de Trabajo, Ambiente y Salud (ISTAS): Paralelo Ediciones S.A.

Moncada, S., Llorens, C., Navarro, A. y Kristensen, T. (2005). ISTAS21: Versión en lengua castellana del cuestionario psicosocial de Copenhague (COPSOQ). Archivos de prevención de riesgos laborales, $8(1), 18-29$.

Moorman, R. (1991). Relationship between organizational justice and organizational citizenship behaviors: Do fairness perceptions influence employee citizenship? Journal of Applied Psychology, 76(6), 845-855.

Mudrak, J., Zabrodska, K., Kveton, P. et ál. (2017). Occupational well-being among university faculty: A job demands-resources model. Research in Higher Education, 59(3), 1-24.

Neffa, J. (2015). Los riesgos psicosociales en el trabajo: contribución a su estudio. Buenos Aires: Universidad Metropolitana para la Educación y el Trabajo. Centro de Innovación para los Trabajadores.

Nunnally, J. \& Bernstein, I. (1994). Psychological theory. New York: McGraw-Hill.

Parker, S., Wall, T. \& Cordery, J. (2001). Future work design research and practice: Towards an elaborated model of work design. Journal of Occupational and Organizational Psychology, 74(4), 413-440.

Pejtersen, J., Kristensen, T., Borg, V. \& Bjorner, J. (2010). The second version of the Copenhagen Psychosocial Questionnaire. Scandinavian Journal of Public Health, 38(3), 8-24.

Piccolo, R., Judge, T., Takahashi, K. et ál. (2005). Core self-evaluations in Japan: Relative effects on job satisfaction, life satisfaction, and happiness. Journal of Organizational Behavior, 26(8), 965-984.

Piedmont, R., McCrae, R., Riemann, R. \& Angleitner, A. (2000). On the invalidity of validity scales: evidence from self-reports and observer ratings in volunteer samples. Journal of Personality and Social Psychology, 78(3), 582-593.

Pujol-Cols, L. J. y Dabos, G. E. (2018). Satisfacción laboral: una revisión de la literatura acerca de sus principales determinantes. Estudios Gerenciales, 34(146), 3-18. 
Rocha, C. \& Chelladurai, P. (2012). Item parcels in structural equation modeling: An applied study in sport management. International Journal of Psychology and Behavioral Sciences, 2(1), 46-53.

Rodríguez, L., Oramas, A. y Rodríguez, E. (2007). Estrés en docentes de educación básica: estudio de caso en Guanajuato, México. Salud de los Trabajadores, 15(1), 5-16.

Rojas, M. \& Rodríguez, J. (2011). Factores de riesgo psicosociales en el profesorado de Enfermería Geriátrica de universidades españolas, Gerokomos, 22(2), 62-71.

Salanova, M. (2008). Organizaciones saludables: una aproximación desde la psicología positiva. En C. Vázquez y G. Hervás (eds.), Psicología positiva aplicada (pp. 403-427). Madrid: Desclée de Brouwer.

Salanova, M., Llorens, S. y García-Renedo, M. (2003). ¿Por qué se están quemando los profesores? Prevención, Trabajo y Salud, 28, 16-20.

Salanova, M., Martínez, I. y Llorens, S. (2005). Psicología organizacional positiva. En F. Palací (coord.), Psicología de la Organización (pp. 349-376). Madrid: Pearson, Prentice-Hall.

Salanova, M., Martínez, I. y Llorens, S. (2014). Una mirada más "positiva” a la salud ocupacional desde la psicología organizacional positiva en tiempos de crisis: aportaciones desde el equipo de investigación WoNT. Papeles del Psicólogo, 35(1), 22-30.

Salanova, M., Martínez, I. y Lorente, L. (2005). ¿Cómo se relacionan los obstáculos y facilitadores organizacionales con el burnout docente? Un estudio longitudinal. Revista de Psicología del Trabajo y de las Organizaciones, 21(1-2), 37-54.

Schaufeli, W. \& Bakker, A. (2004). Job demands, job resources, and their relationship with burnout and engagement: A multi-sample study. Journal of Organizational Behavior, 25(3), 293-315.

Schaufeli, W. y Salanova, M. (2002). ¿Cómo evaluar los riesgos psicosociales en el trabajo? Prevención, Trabajo y Salud, 20(1), 4-9.

Schaufeli, W., Salanova, M., González-Romá, V. \& Bakker, A. (2002). The measurement of engagement and burnout: A two sample confirmatory factor analytic approach. Journal of Happiness Studies, 3(1), 71-92.

Seligman, M. \& Csikszentmihalyi, M. (2000). Positive psychology: An introduction American Psychologist, 55(1), 5-14.

Shockley, K. \& Allen, T. (2007). When flexibility helps: Another look at the availability of flexible work arrangements and work-family conflict. Journal of Vocational Behavior, 71(3), 479-493.

Siegrist, J. (1996). Adverse health effects of high-effort/low-reward conditions. Journal of Occupational Health Psychology, 1(1), 27-41.

Tavakol, M. \& Dennick, R. (2011). Making sense of Cronbach's alpha. International Journal of Medical Education, 2, 53-55. 
Unda, S., Sandoval, J. y Gil-Monte, P.R. (2007). Prevalencia del síndrome de quemarse por el trabajo (SQT) (burnout) en maestros mexicanos. Información Psicológica, 91, 53-63.

Unda, S., Uribe, F., Jurado, S., García, M., Tovalín, H. y Juárez, A. (2016). Elaboración de una escala para valorar los factores de riesgo psicosocial en el trabajo de profesores universitarios. Revista de Psicología del Trabajo y de las Organizaciones, 32(2), 67-74.

Van Veldhoven, M., Meijman, T., Broersen, J. \& Fortuin, R. (1997). Handleiding VBBA: Onderzoek naar de beleving van psychosociale arbeidsbelasting en werkstress met behulp van de vragenlijst beleving en beoordeling van de arbeid. Amsterdam: SKB Vragenlijst Services.

Watts, J. \& Robertson, N. (2011). Burnout in university teaching staff: A systematic literature review. Educational Research, 53(1), 33-50.

Xanthopoulou, D., Bakker, A., Demerouti, E. \& Schaufeli, W. (2007). The role of personal resources in the job demands-resources model. International Journal of Stress Management, 14(2), 121-141. 